\title{
2020 vision
}

This year will see the finalization of a global framework for biodiversity for the next decade and beyond. We look at the reasons to be optimistic and call for increased engagement in the process.

T he Convention on Biological Diversity (CBD) came into existence in 1992, and more recently produced a strategic plan for biodiversity in 2010 to cover the decade 2011-2020. In 2020, a new framework for global biodiversity will be set out, the result of assessments and discussions that are already in full swing. This framework will set the biodiversity agenda for the next decade and beyond, towards the 2050 vision of 'Living in harmony with nature'. A lot has changed since 1992, and indeed since 2010, for better and for worse, in terms of policy, public perception and the state of biodiversity itself.

It is probably necessary to start with the negatives. The majority of the Aichi targets set in 2010 are unlikely to be met, deforestation shows little sign of any consistent and sustained slowing down, the critical state of coral reefs continues to deteriorate rapidly, and carbon emissions continue to rise. It would probably also be fair to say that the political climate on environmental issues has become less favourable, certainly in some key countries such as Brazil and the United States.

But there are also changes that allow us to be cautiously optimistic. The CBD no longer stands alone as the international face of biodiversity policy. The Intergovernmental Science-Policy Platform on Biodiversity and Ecosystem Services (IPBES) published its first global assessment in 2019, providing a prominent statement of environmental priorities. The UN Sustainable Development Goals are gaining traction in policy discussions at all levels, and embed biodiversity in the wider context of social and economic strategies. Related to this, biodiversity is starting to become better integrated into other environmental efforts, such as the recognition of nature-based solutions for climate mitigation or the importance of biodiversity for food security and health. There is now a much deeper appreciation of the importance of stakeholder diversity in environmental policymaking, for example in terms of gender, geography and traditional knowledge, which strengthens outcomes. On top of these developments, public recognition of the importance of environmental issues in general, and biodiversity in particular, continues to rise.

There has also been progress on some specific issues. Protected area designation, in both marine and terrestrial realms, is rising, even if we should not be blindly over-confident about effectiveness.

The IPBES's first major report was on pollinators, and trans-national policies such as the EU's neonicotinoid ban and smaller-scale policies for pollinatorfriendly habitats in cities demonstrate how seriously this issue is being taken. Current discussions about agricultural reforms, such as an update to the EU's much-criticized Common Agricultural Policy and a possible post-Brexit policy in the United Kingdom, suggest that nature protection will be valued more than before. And 'payments for ecosystem services' is an approach that has seen success in various regions.

This combination of specific progress and increased engagement suggests that there is a great opportunity to be seized with the post-2020 framework. The process is already well under way, with inter-sessional working groups established at previous meetings and a draft document to be commented on in the first half of the year. The landscape of feeder meetings and science and policy stakeholders is complex, and confusing even to the initiated. Compromise, personality and political developments way beyond the control of biodiversity professionals will all play a role. For many researchers, these policy discussions will feel a world away from their on-the-ground experience of both conservation successes and failures. Yet, most such researchers are only a few connections away from someone who is directly engaged and who can channel ideas and concerns. As a journal, we hope to provide a forum for stakeholders to engage with each other as they hone the final framework, and for the whole research community to keep engaged with the process. As we approach the final phase, and commence the 'super year of biodiversity', there is still much to play for.

Published online: 3 January 2020 https://doi.org/10.1038/s41559-019-1083-z 